\title{
A NOTE ON ALTERNATING SERIES IN SEVERAL DIMENSIONS
}

\author{
DAVID BORWEIN \\ Department of Mathematics, The University of Western Ontario, London, Ontario, Canada N6A $5 B 7$
}

\author{
JONATHAN M. BORWEIN \\ Department of Mathematics, Statistics and Computing Science, Dalhousie University, Halifax, Nova Scotia, \\ Canada $\mathrm{B} 3 \mathrm{H} 3 \mathrm{~J} 5$
}

1. Introduction. In the course of a study of chemical lattice sums.[1] the authors considered sums such as

$$
\sum^{\prime}(-1)^{n+m+k}\left(n^{2}+m^{2}+k^{2}\right)^{-1 / 2},
$$

the summation being over all non-zero integer triples. Such "sums" occur naturally in the study of crystal potentials. For example, (1.1) is meant to measure the potential at the origin of an infinite cubic crystal with unit Coulomb charges at each integer lattice point. As such the sum is considered to represent an electrochemical constant (Madelung's constant) for sodium chloride. An excellent account of such lattice sums can be found in Glasser and Zucker's recent survey [3].

The series in (1.1) is not absolutely convergent and hence its sum is not order independent. Various possible orders suggest themselves. The chemical literature is somewhat vague on this point. As discussed in [1], it is really only appropriate to consider rectangular sums.

We shall consider alternating series of the form

$$
\sum(-1)^{\bar{m}} f(\bar{m})
$$

where $\bar{m}=\left(m_{1}, m_{2}, \ldots, m_{N}\right)$ ranges over $\mathbb{N}^{N}$, the $N$-fold product of non-negative integers, $(-1)^{\bar{m}}:=(-1)^{m_{1}+m_{2}+\cdots+m_{N}}$ and $f: \mathbb{N}^{N} \rightarrow \mathbb{R}$. For $s: \mathbb{N}^{N} \rightarrow \mathbb{R}, s(\bar{n})$ is said to converge to a limit l as $\bar{n}$ increases in $\mathbb{N}^{N}$ if, given $\varepsilon>0$, there is an $\bar{m}$ in $\mathbb{N}^{N}$ such that

$$
|s(\bar{n})-l|<\varepsilon \text { whenever } \bar{n} \geqslant \bar{m},
$$

the notation $\bar{n} \geqslant \bar{m}$ or $\bar{m} \leqslant \bar{n}$ meaning that $n_{i} \leqslant m_{i}$ for $i=1,2, \ldots, N$. This is equivalent to convergence in the sense of Pringsheim, which requires that $s(\bar{n}) \rightarrow l$ as $\min _{1 \leqslant i \leqslant N} n_{i} \rightarrow \infty$. For discussion of various concepts of convergence, see [2], [4], [5], [6], [7] and the references therein. We shall show that the sum of (1.2) exists under appropriate conditions if it is defined as the limit

David Borwein was born in Lithuania and raised in South Africa. He received a B.Sc. in electrical engineering from the University of Witwatersrand in 1945 and a B.Sc. in mathematics from that university in 1948 . In 1950 he obtained a Ph.D. in mathematics from University College, London, England (under the supervision of Stephen Bosanquet) and a D.Sc. from the same university in 1960. In 1959 he was elected a Fellow of the Royal Society of Edinburgh. He taught in St. Andrews, Scotland, for thirteen years before moving to The University of Western Ontario, London, Canada, where he has served as Professor since 1963 and Head of the Department of Mathematics since 1967. He has written extensively on summability theory and other areas of analysis. He has served as Advanced Problems Editor for the Monthly and is President of the Canadian Mathematical Society. His hobbies include bridge and jogging. In addition to supervising eleven doctoral students, he has also reared two mathematicians: his sons Peter and Jonathan.

Jonathan Borwein was reared in Scotland and Canada. In 1971 he completed a B.Sc. in mathematics at The University of Western Ontario and won a Rhodes Scholarship to Oxford University where he obtained a D.Phil. under the supervision of M.A.H. Demptster in 1974. Since then he has taught at Dalhousie University, Halifax, Canada, where he is a Professor of Mathematics. He was also on the faculty at Carnegie-Mellon from 1980 to 1982. His principal research interests are optimization theory, analysis, and functional analysis. With his brother he has just completed a book on Analytic Computation Theory. $\mathrm{He}$ is an ex-competitive bridge player, a distance swimmer, and an election organizer.

This research was partially supported by the Natural Sciences and Engineering Research Council of Canada, and completed while the second author was visiting the Control and Management Systems Division of Cambridge University. 
as $\bar{n}$ increases in $\mathbb{N}^{N}$ of

$$
\sum_{\overline{0} \leqslant \bar{m} \leqslant \bar{n}}(-1)^{\bar{m}} f(\bar{m})
$$

Our main result, Theorem 3.1, is a complete generalization to multiple series of the classical result due to Leibniz, according to which if $\left\{a_{n}\right\}$ is a monotonic sequence of non-negative real numbers converging to zero, then the series $\sum_{n=0}^{\infty}(-1)^{n} a_{n}$ converges to $s$ and its sequence of partial sums $\left\{s_{n}\right\}$ satisfies

$$
0 \leqslant s_{2 m+1} \leqslant s_{2 m+3} \leqslant s \leqslant s_{2 n+2} \leqslant s_{2 n} \text { for all } m, n \text { in } \mathbb{N} .
$$

Theorem 3.1 exhibits order relations between partial sums of the form (1.3) which hold when the function $f$ is fully monotone (as defined in $\S 2$ below); and Lemma 3.1, which plays a key role in the proof of Theorem 3.1, gives precise bounds on the size of these partial sums and also on the difference between them and their limit when it exists (see (3.6) below). To our knowledge neither such order nor such bound results have been considered previously in more than one dimension. There are, however, results available guaranteeing the convergence of non-absolutely convergent multiple dimensional series. Hardy [4] derives a "bounded convergence" test based on Abel partial summation which can be used to establish convergence criteria for series like (1.2) (see also Móricz [6]). Bromwich [2, p. 97] discusses the 2-dimensional version of Hardy's result. Meyer [5] gives necessary and sufficient conditions for the "diagonal summability" of 2-dimensional monotonic alternating series.

When $f$ is the restriction of an $N$-times continuously differentiable function, a most satisfactory test involving partial derivatives is given by our Theorem 4.1 for the convergence of the alternating series (1.2). This theorem follows immediately from our Lemma 2.1 which has independent interest as a mean value estimate for general alternating sums.

We prove all our results for $N$-dimensions. The interested reader will be able to provide much simpler arguments for the 2-dimensional case as indicated pictorially in $\S 3$ below. The 3-dimensional case, which is of primary interest, is not substantially simpler than the general one. Our vector notation enables us to express $N$-dimensional results concisely. An explicit treatment of the series (1.1) and its 2-dimensional analogue is given in [1].

2. Preliminaries. Let $\mathbb{N}^{N}, \mathbb{Z}^{N}, \mathbb{P}^{N}, \mathbb{R}^{N}$ denote respectively the $N$-fold product of the non-negative integers, the integers, the non-negative real numbers, and the real numbers. We denote by $\overline{1}$ the vector in $\mathbb{R}^{N}$ with every component 1 , and by $\bar{e}_{j}$ the vector with $j$ th component 1 and every other component 0 . For

$$
\bar{a}=\left(a_{1}, a_{2}, \ldots, a_{N}\right), \bar{b}=\left(b_{1}, b_{2}, \ldots, b_{N}\right) \text { in } \mathbb{R}^{N},
$$

we define

$$
|\bar{a}|:=\max _{1 \leqslant i \leqslant n}\left|a_{1}\right|, \quad \bar{a} \cdot \bar{b}:=a_{1} b_{1}+a_{2} b_{2}+\cdots+a_{n} b_{n}, \quad \bar{a} \bar{b}:=\left(a_{1} b_{1}, a_{2} b_{2}, \ldots, a_{N} b_{N}\right) ;
$$

and for $\bar{s}=\left(s_{1}, s_{2}, \ldots, s_{N}\right)$ in $\mathbb{Z}^{N}$, we define

$$
(-1)^{\bar{s}}:=(-1)^{\overline{1} \cdot \bar{s}}=(-1)^{s_{1}+s_{2}+\cdots+s_{N}} \text {. }
$$

A function $f: \mathbb{N}^{N} \rightarrow \mathbb{R}$ is said to be $(N-)$ monotone (decreasing) if

$$
\sum_{\substack{|\bar{s}| \leqslant 1 \\ \bar{s} \in \mathbb{N}^{N}}}(-1)^{\bar{s}} f(\bar{m}+\bar{s}) \geqslant 0
$$

for all $\bar{m}$ in $\mathbb{N}^{N}$. We shall say that $f$ is fully monotone if $f$ and all its coordinate restrictions are monotone. Then 1-monotonicity means that $f(m) \geqslant f(m+1)$ for $m$ in $\mathbb{N}$; 2-monotonicity requires that 


$$
f(m, n)+f(m+1, n+1) \geqslant f(m, n+1)+f(m+1, n) \text { for } m, n \text { in } \mathbb{N}
$$

and in general $N$-monotonicity of $f$ requires the alternating sum (1.2) over any unit $N$-cube to have the same sign as $(-1)^{\bar{m}}$ at the corner nearest the origin. Note that any linear functional $\Phi$ on $\mathbb{R}^{N}$ is $N$-monotone for $N>1$, but $\Phi$ is fully monotone only if $\Phi \leqslant 0$ on $\mathbb{P}^{N}$.

We prove a lemma useful for obtaining criteria for monotonicity in which subscripts denote partial derivatives taken in order of the subscripts and

$$
\mathbb{P}_{*}^{N}:=\left\{\bar{x} \in \mathbb{P}^{N} \mid x_{\imath}>0 \quad \text { for } i=1,2, \ldots, N\right\}
$$

Lemma 2.1. (a) Let the function $f: \mathbb{P}_{*}^{N} \rightarrow \mathbb{R}$ have partial derivatives $f_{12 \ldots N}$ throughout $\mathbb{P}_{*}^{N}$. Then, for $\bar{x} \in \mathbb{P}_{*}^{N}, \bar{a} \in \mathbb{P}^{N}$,

$$
\sum_{\substack{|\bar{s}| \leqslant 1 \\ \bar{s} \in \mathbb{N}^{N}}}(-1)^{\bar{s}} f(\bar{x}+\bar{a} \bar{s})=(-1)^{N} a_{1} a_{2} \cdots a_{N} f_{12 \ldots N}(\bar{c})
$$

for some $\bar{c}$ between $\bar{x}$ and $\bar{x}+\bar{a}$.

(b) Let the function $\psi: \mathbb{P}_{*} \rightarrow \mathbb{R}$ be $N$-times differentiable. Then, for $x>0, \bar{a} \in \mathbb{P}^{N}$,

$$
\sum_{\substack{|\bar{s}| \leqslant 1 \\ \bar{s} \in \mathbb{N}^{N}}}(-1)^{\bar{s}} \psi(x+\bar{a} \cdot \bar{s})=(-1)^{N} a_{1} a_{2} \cdots a_{N} \psi^{(N)}(c)
$$

for some c between $x$ and $x+a_{1}+a_{2}+\cdots+a_{N}$.

Proof. (a) The result is true for $N=1$. Suppose inductively that it is true with $N-1$ in place of $N$. The left-hand side of (2.2) is evidently equal to

$$
\sum_{\substack{|s| \leq 1 \\ \bar{s} \in \mathbb{N}^{N-1}}}(-1)^{\bar{s}} g\left(x_{1}+a_{1} s_{1}, x_{2}+a_{2} s_{2}, \ldots, x_{N-1}+a_{N-1} s_{N-1}\right),
$$

where $g: \mathbb{P}_{*}^{N-1} \rightarrow \mathbb{R}$ is defined by

$$
g(\bar{t}):=f\left(\bar{t}, x_{N}\right)-f\left(\bar{t}, x_{N}+a_{N}\right) .
$$

Hence, by the inductive hypothesis and the mean value theorem, the left-hand side of (2.2) equals

$$
\begin{gathered}
(-1)^{N-1} a_{1} a_{2} \cdots a_{N-1} g_{12 \ldots N-1}\left(c_{1}, c_{2}, \ldots, c_{N-1}\right) \\
=(-1)^{N} a_{1} a_{2} \cdots a_{N} f_{12 \ldots N}\left(c_{1}, c_{2}, \ldots, c_{N}\right),
\end{gathered}
$$

where $x_{\imath} \leqslant c_{\imath} \leqslant x_{i}+a_{\imath}$ for $i=1,2, \ldots, N$.

(b) Define $f: \mathbb{P}_{*}^{N} \rightarrow \mathbb{R}$ by $f(\bar{t}):=\psi\left(t_{1}+t_{2}+\cdots+t_{N}\right)$. Then, with $\bar{x}:=(x, x, \ldots, x) / N$, we have

$$
f(\bar{x}+\bar{a} \bar{s})=\psi(x+\bar{a} \cdot \bar{s})
$$

and (2.3) follows from (2.2).

The following lemma yields a stock of fully monotone functions.

LEMMA 2.2. Let the function $\psi: \mathbb{P} \rightarrow \mathbb{R}$ be continuous and satisfy

$$
(-1)^{n} \psi^{(n)}(x) \geqslant 0 \text { for all } x>0 \text { and } n=1,2, \ldots, N \text {. }
$$

Then

(a) for $x \geqslant 0, \bar{a} \in \mathbb{P}^{N}$,

$$
\sum_{\substack{|\bar{s}| \leqslant 1 \\ \bar{s} \in \mathbb{N}^{N}}}(-1)^{\bar{s}} \psi(x+\bar{a} \cdot \bar{s}) \geqslant 0
$$



by

(b) given non-decreasing functions $g_{i}: \mathbb{N} \rightarrow \mathbb{N}, i=1,2, \ldots, N$, the function $f: \mathbb{N}^{N} \rightarrow \mathbb{R}$ defined

$$
f(\bar{m}):=\psi\left(\sum_{i=1}^{N} g_{\imath}\left(m_{\imath}\right)\right)
$$

is fully monotone on $\mathbb{N}^{N}$.

Proof. (a) It follows from (2.3) that (2.5) holds for $x>0$. By continuity it also holds for $x=0$.

(b) We have

$$
\sum_{|\bar{s}| \leqslant 1}(-1)^{\bar{s}} f(\bar{m}+\bar{s})=\sum_{|\bar{s}| \leqslant 1}(-1)^{\bar{s}} \psi\left(\sum_{l=1}^{N} g_{l}\left(m_{t}\right)+\sum_{l=1}^{N} s_{l}\left(g_{l}\left(m_{i}+1\right)-g_{i}\left(m_{l}\right)\right)\right) \geqslant 0
$$

by part (a), since $g_{i}\left(m_{l}+1\right)-g_{i}\left(m_{l}\right) \geqslant 0$ and $\sum_{t=1}^{N} g_{l}\left(m_{i}\right) \geqslant 0$. Thus $f$ in $N$-monotone. Since the argument applies with some of the $g_{\imath}$ constant, it follows that $f$ is in fact fully monotone.

EXAMPLE 2.1. (a) Let

$$
\|\bar{m}\|_{p}:=\left(\sum_{l=1}^{N}\left|m_{l}\right|^{p}\right)^{1 / p} \quad(p>0)
$$

Then

$$
f(\bar{m}):=\|\overline{1}+\bar{m}\|_{p}^{-q} \quad(q>0)
$$

is fully monotone, by Lemma $2.2(\mathrm{~b})$ with

$$
\psi(x):=(N+x)^{-q / p}
$$

and each $g_{l}(x):=(1+x)^{p}-1$. Note that with $\|\bar{m}\|_{\infty}:=|\bar{m}|$ the function $f$ is still fully monotone for $p=\infty$.

(b) Similarly the functions

$$
f(\bar{m}):=-\log \left(\sum_{\imath=1}^{N} e^{a_{\imath} m_{\imath}}\right) \quad\left(a_{\imath}>0\right)
$$

and

$$
f(\bar{m}):=\prod_{i=1}^{N}\left(1+m_{l}\right)^{-1 / N}=\exp \left(-\frac{1}{N} \sum_{i=1}^{N} \log \left(1+m_{l}\right)\right)
$$

are fully monotone.

3. Alternating sums over rectangles. Before proceeding to the main results it is convenient to prove the following lemma.

LEMMA 3.1. Let $f: \mathbb{N}^{N} \rightarrow \mathbb{P}$ be fully monotone. Then

$$
0 \leqslant(-1)^{\bar{a}} \sum_{\bar{a} \leqslant \bar{m} \leqslant \bar{n}}(-1)^{\bar{m}} f(\bar{m}) \leqslant f(\bar{a})
$$

whenever $\bar{a}, \bar{n} \in \mathbb{N}^{N}$ and $\bar{a} \leqslant \bar{n}$.

Proof. It suffices to prove (3.1) for the case $\bar{a}=\overline{0}$, since the general case follows from this case with $f(\bar{m})$ replaced by $f(\bar{m}+\bar{a})$. We establish the first inequality in (3.1) by induction. Clearly it holds for $N=1$. Suppose it holds with $N-1$ in place of $N$. Observe that, for $\bar{n}=\left(n_{1}, n_{2}, \ldots, n_{N}\right) \in \mathbb{N}^{N}$, 


$$
\sum_{\overline{0} \leqslant \bar{m} \leqslant \bar{n}}(-1)^{\bar{m}} f(\bar{m})=S_{1}+S_{2}
$$

with

$$
\begin{aligned}
S_{1} & :=\sum_{\overline{0} \leqslant 2 \bar{k} \leqslant \bar{n}}(-1)^{2 \bar{k}} \sum_{\overline{0} \leqslant \bar{s} \leqslant \overline{1}}(-1)^{\bar{s}} f(2 \bar{k}+\bar{s}), \\
S_{2} & :=\sum_{i \in E} \sum_{\substack{m_{i}=n_{i} \\
\overline{0} \leqslant \bar{m} \leqslant \bar{a}_{i}}}(-1)^{\bar{m}} f(\bar{m}),
\end{aligned}
$$

where $E:=\left\{i \mid n_{i}\right.$ even $\}$, and $\bar{a}_{i}:=\left(\alpha_{1}, \alpha_{2}, \ldots, \alpha_{N}\right)$ with

$$
\alpha_{j}:= \begin{cases}n_{j}-1 & \text { if } j<i, j \in E \text { and } \quad n_{j} \neq 0, \\ n_{j} & \text { otherwise. }\end{cases}
$$

Now $S_{1} \geqslant 0$ because of the $N$-monotonicity of $f$ and $S_{2} \geqslant 0$ by the inductive hypothesis. The validity of the first inequality in (3.1) for every $N \geqslant 1$ follows.

To establish the second inequality in (3.1), we observe that

$$
\sum_{\overline{0} \leqslant \bar{m} \leqslant \bar{n}}(-1)^{\bar{m}} f(\bar{m})-f(\overline{0})=-\sum_{\substack{0 \\ \bar{s} \neq \bar{s} \leqslant 1}}(-1)^{\bar{s}} \sum_{\bar{s} \leqslant \bar{m} \leqslant \bar{n}}(-1)^{\bar{m}} f(\bar{m}) \leqslant 0
$$

by the first inequality in (3.1).

In what follows we use the notation:

$$
t_{\bar{n}}:=t_{\bar{n}}(f):=\sum_{\overline{0} \leqslant \bar{m} \leqslant \bar{n}}(-1)^{\bar{m}} f(\bar{m}),
$$

where $\bar{m}, \bar{n} \in \mathbb{N}^{N}$.

THEOREM 3.1 (Alternating series test). If $f: \mathbb{N}^{N} \rightarrow \mathbb{P}$ is fully monotone, $\bar{n}, \bar{r} \in \mathbb{N}^{N}$ and $\bar{n} \leqslant \bar{r}$, then

(i) $t_{2 \bar{n}} \geqslant t_{2 \bar{r}} \geqslant 0$

(ii) $t_{2 \bar{r}+\overline{1}} \geqslant t_{2 \bar{n}+\overline{1}} \geqslant 0$,

(iii) $t_{2 \bar{n}} \geqslant t_{2 \bar{n}+\overline{1}}$.

If in addition $\lim _{n \rightarrow \infty} f\left(n \bar{e}_{i}\right)=0$ for $i=1,2, \ldots, N$, then $t_{\bar{n}}$ converges to a limit $t$ as $\bar{n}$ increases, $t_{2 \bar{n}} \geqslant t \geqslant t_{2 \bar{n}+\overline{1}}$ for each $\bar{n} \in \mathbb{N}^{N}$, and consequently

$$
t=\sum_{m_{1}=0}^{\infty} \sum_{m_{2}=0}^{\infty} \cdots \sum_{m_{N}=0}^{\infty}(-1)^{\bar{m}} f(\bar{m})
$$

Proof. To establish (i) and (ii) it suffices to consider $\bar{r}:=\bar{n}+\bar{e}_{j}, 1 \leqslant j \leqslant N$. We then have

$$
t_{2 \bar{r}}-t_{2 \bar{n}}=\sum_{\bar{a} \leqslant \bar{m} \leqslant 2 \bar{r}}(-1)^{\bar{m}} f(\bar{m}) \quad \text { with } \bar{a}:=\left(2 n_{j}+1\right) \bar{e}_{j},
$$

and

$$
t_{2 \bar{r}+\overline{1}}-t_{2 \bar{n}+\overline{1}}=\sum_{\bar{b} \leqslant \bar{m} \leqslant 2 \bar{r}+\overline{1}}(-1)^{\bar{m}} f(\bar{m}) \quad \text { with } \bar{b}:=\left(2 n_{j}+2\right) \bar{e}_{j} .
$$

Since $(-1)^{\bar{a}}=-1$ and $(-1)^{\bar{b}}=1$, (i) and (ii) follow by Lemma 3.1. Next, by an "inclusionexclusion" counting argument, we have

$$
t_{2 \bar{n}+\overline{1}}-t_{2 \bar{n}}=-\sum_{i=1}^{2^{N}-1}(-1)^{\bar{a}_{i}} \sum_{\bar{a}_{i} \leqslant \bar{m} \leqslant 2 \bar{n}+\overline{1}}(-1)^{\bar{m}} f(\bar{m}),
$$


each $\bar{a}_{t}$ being of the form $\left(\alpha_{1}, \alpha_{2}, \ldots, \alpha_{N}\right) \neq \overline{0}$ with every $\alpha_{j}$ either 0 or $2 n_{j}+1$. Lemma 3.1 now yields (iii).

It follows from (i) that $t_{2 \bar{n}}$ converges (to $\inf _{\bar{n} \in \mathbb{N}^{N}} t_{2 \bar{n}}$ ) as $\bar{n}$ increases in $\mathbb{N}^{N}$. Hence to establish the convergence of $t_{\bar{n}}$ as $\bar{n}$ increases in $\mathbb{N}^{N}$, it suffices to show that, for fixed $\bar{k}$ in $\mathbb{N}^{N}, t_{\bar{n}+\bar{k}}-t_{\bar{n}}$ $\rightarrow 0$ as $\bar{n}$ increases, and for this it is enough to consider $\bar{k}:=\bar{e}_{j}$. We then have

$$
t_{\bar{n}+\bar{k}}-t_{\bar{n}}=\sum_{\bar{c} \leqslant \bar{m} \leqslant \bar{n}+\bar{k}}(-1)^{\bar{m}} f(\bar{m}) \quad \text { with } \bar{c}:=\left(n_{j}+1\right) \bar{e}_{j},
$$

and hence, by Lemma 3.1,

$$
\left|t_{\bar{n}+\bar{k}}-t_{\bar{n}}\right| \leqslant f(\bar{c})
$$

which tends to 0 as $\bar{n}$ increases.

Since $f$ is fully monotone, an inductive argument shows that the sums implicit in (3.3) exist and that the identity holds.

The convergence conclusion also follows from Hardy's "bounded series" test which also requires full monotonicity without so naming it. Hardy's result, however, does not yield the alternation information expressed by (i), (ii) and (iii).

To indicate the underlying geometric simplicity of our proof we show pictorially why (i), (ii) and (iii) hold in the two-dimensional case when $\bar{n}=\left(n_{1}, n_{2}\right)$ and $\bar{r}=\left(n_{1}+1, n_{2}\right)$.

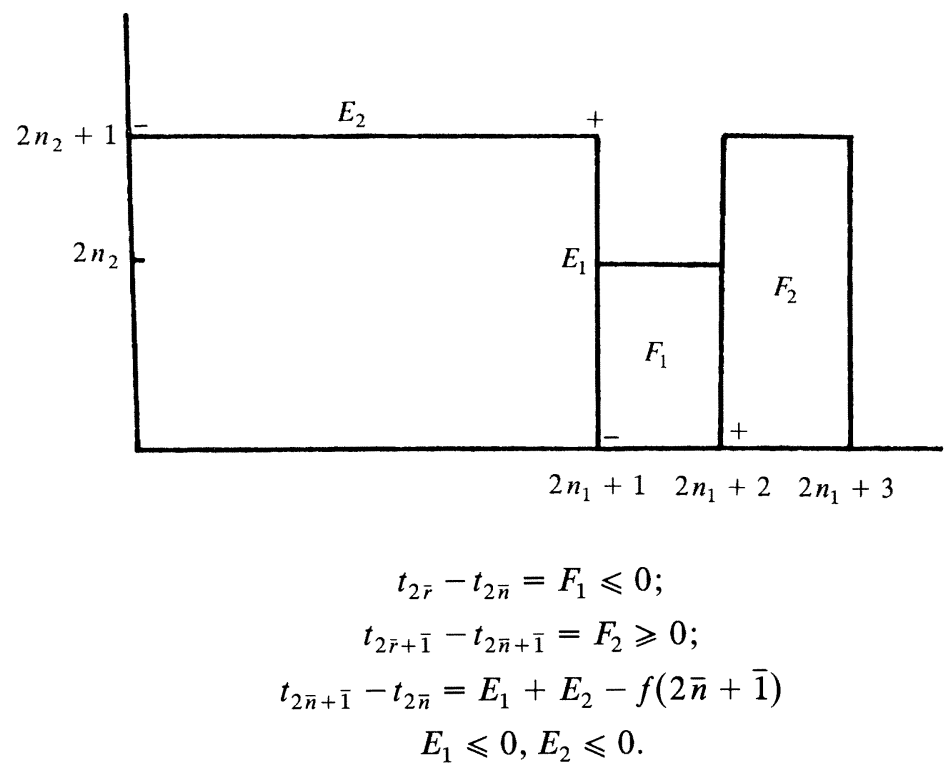

Here $F_{l}$ represents the sum over the enclosed rectangle and $E_{i}$ the sum over the adjacent edge.

The next theorem is a special case of Theorem 3.1. We use the notation:

$$
t_{n}:=t_{n}(f):=\sum_{|\bar{m}| \leqslant n}(-1)^{\bar{m}} f(\bar{m})
$$

where $\bar{m} \in \mathbb{N}^{N}$ and $n \in \mathbb{N}$. Observe that $t_{n}=t_{n \overline{1}}$.

THEOREM 3.2. If $f: \mathbb{N}^{N} \rightarrow \mathbb{P}$ is fully monotone, then, for $m, n \in \mathbb{N}$,

$$
0 \leqslant t_{2 m+1} \leqslant t_{2 m+3} \leqslant t_{2 n+2} \leqslant t_{2 n} \text {. }
$$

If in addition $\lim _{n \rightarrow \infty} f\left(n \bar{e}_{i}\right)=0$ for $i=1,2, \ldots, N$, then $t_{n}$ converges to a limit $t$ as $n \rightarrow \infty$, and $t_{2 n} \geqslant t \geqslant t_{2 n+1} \stackrel{n \rightarrow \infty}{\text { for each }} n \in \mathbb{N}$ 
Corollary 3.3. For $0<p \leqslant \infty, q>0, \bar{n} \in \mathbb{N}^{N}$,

$$
S_{\bar{n}}^{N}:=\sum_{\overline{1} \leqslant \bar{m} \leqslant \bar{n}}(-1)^{\bar{m}}\|\bar{m}\|_{p}^{-q}
$$

converges as $\bar{n}$ increases.

Proof. We have

$$
S_{\bar{n}+\overline{1}}^{N}=(-1)^{N} \sum_{\overline{0} \leqslant \bar{m} \leqslant \bar{n}}(-1)^{\bar{m}}\|\overline{1}+\bar{m}\|_{p}^{-q}
$$

and Theorem 3.1 applied to Example 2.1 (a) yields the required convergence.

Corollary 3.4. For $0<p \leqslant \infty, q>0, \bar{n} \in \mathbb{N}^{N}$,

$$
A_{\bar{n}}^{N}:=\sum_{\substack{-\bar{n} \leqslant \bar{m} \leqslant \bar{n} \\ \bar{m} \in \mathbb{Z}^{N} \backslash\{\overline{0}\}}}(-1)^{\bar{m}}\|\bar{m}\|_{p}^{-q}
$$

converges as $\bar{n}$ increases.

Proof. Observe that

$$
A_{\bar{n}}^{N}=2^{N} S_{\bar{n}}^{N}+R
$$

where $R$ is the sum of a number of finite series each of the same general form as $A_{\bar{n}}^{N}$ but of lower dimension. The desired result follows by induction.

Similarly we have:

COROllaRY 3.5. For $0<p \leqslant \infty, q>0, \bar{a} \in \mathbb{R}^{N}, \bar{n} \in \mathbb{N}^{N}$,

$$
\sum_{\substack{-\bar{n} \leqslant \bar{m} \leqslant \bar{n} \\ \bar{m} \in \mathbb{Z}^{N} \backslash\{\overline{0}\}}}(-1)^{\bar{m}}\|\bar{a} \bar{m}\|_{p}^{-q}
$$

converges as $\bar{n}$ increases.

In particular Madelung's constant exists for any rectilinear lattice in $\mathbb{R}^{N}$ if defined as

$$
M^{N}(\bar{a}):=\lim _{n \rightarrow \infty} \sum_{\substack{|\bar{m}| \leqslant n \\ \bar{m} \in \mathbb{Z}^{N} \backslash\{\overline{0}\}}}(-1)^{\bar{m}}\|\bar{a} \bar{m}\|_{2}^{-1} .
$$

By virtue of the underlying alternation it is easy to obtain a good error bound for $t-t_{\bar{n}}$, when $t, t_{\bar{n}}$ are as in Theorem 3.1. If $f: \mathbb{N}^{N} \rightarrow \mathbb{R}$ is fully monotone and $t_{\bar{n}}$ converges to $t$ as $\bar{n}$ increases, then, letting $\bar{n}_{0}:=\bar{n}$ and $\bar{n}_{j}:=\bar{n}_{j-1}+\bar{e}_{j}$ for $j=1,2, \ldots, N$, we have

$$
\left|t-t_{\bar{n}}\right| \leqslant\left|t_{\bar{n}+\overline{1}}-t_{\bar{n}}\right| \leqslant \sum_{j=1}^{N}\left|t_{\bar{n}_{j}}-t_{\bar{n}_{j-1}}\right| \leqslant \sum_{j=1}^{N} f\left(\left(n_{j}+1\right) \bar{e}_{j}\right),
$$

on repeated application of (3.4). For the series in (1.1) the difference between

$$
\sum_{\substack{|\bar{m}| \leqslant n \\ \bar{m} \in \mathbb{Z}^{N} \backslash\{\overline{0}\}}}(-1)^{\bar{m}}\|\bar{m}\|_{2}^{-1}
$$

and the limit is at most $N /(n+1)$. Thus, to compute 15 digits of Madelung's constant for $\mathrm{NaCl}$ directly would appear to take around $10^{45}$ calculations! No wonder indirect transform techniques are used in practice [3]. Actually only a few digits seem to be used in applications. The $\mathrm{NaCl}$ crystal would have to be galaxy sized for a 15 digit approximation to have physical significance. This indicates the limited utility of using an infinite model for a finite crystal.

By virtue of Example 2.1 (b) and Theorem 3.1, 


$$
\sum_{\overline{1} \leqslant \bar{m} \leqslant \bar{n}}(-1)^{\bar{m}}\left(\prod_{i=1}^{N} m_{l}\right)^{-1 / N}
$$

converges as $\bar{n}$ increases in $\mathbb{N}^{N}$. This is the limiting case as $p \rightarrow 0$ of Corollary 3.3 with $q=1$. In fact the sum is just

$$
\left(\sum_{m=1}^{\infty}(-1)^{m} m^{-1 / N}\right)^{N} .
$$

More generally, in view of Lemma 2.2, Theorem 3.1 applies to $f(\bar{m}):=\prod_{i=1}^{N} g_{i}\left(m_{i}\right)$ whenever each $g_{i}: \mathbb{N} \rightarrow \mathbb{N}$ decreases to zero. In this case, of course,

$$
\sum_{\overline{0} \leqslant \bar{m} \leqslant \bar{n}}(-1)^{\bar{m}} \prod_{i=1}^{N} g_{i}\left(m_{i}\right)
$$

converges to

$$
\prod_{i=1}^{N} \sum_{m=0}^{\infty}(-1)^{m} g_{i}(m)
$$

as $\bar{n}$ increases in $\mathbb{N}^{N}$.

4. A characterization involving partial derivatives. We define a function $f: \mathbb{P}^{N} \rightarrow R$ to be $(\mathrm{N}$-) monotone if

$$
\sum_{\substack{|\bar{s}| \leqslant 1 \\ \bar{s} \in \mathbb{N}^{N}}}(-1)^{\bar{s}} f(\bar{x}+\bar{a} \bar{s}) \geqslant 0
$$

whenever $\bar{x}, \bar{a} \in \mathbb{P}^{N}$. We shall say that $f$ is fully monotone on $\mathbb{P}^{N}$ if $f$ and all its coordinate restrictions are monotone. Then Lemma 2.2 (b) has an obvious analogue. More importantly, we have the following characterization of full monotonicity on $\mathbb{P}^{N}$ in which subscripts denote partial derivatives.

THEOREM 4.1. Let $f: \mathbb{P}^{N} \rightarrow \mathbb{R}$ have continuous partial derivatives of order $N$. Then

(a) $f$ is monotone on $\mathbb{P}^{N}$ if and only if $(-1)^{N} f_{12 \ldots N} \geqslant 0$, and

(b) $f$ is fully monotone on $\mathbb{P}^{N}$ if and only if, for $1 \leqslant k \leqslant N$,

$$
(-1)^{k} f_{l_{1} i_{2} \ldots i_{k}} \geqslant 0
$$

whenever $i_{1}, i_{2}, \ldots, i_{k}$ are distinct integers in $\{1,2, \ldots, N\}$.

Proof. (a) By Lemma 2.1 (a), for $\bar{x} \in \mathbb{P}_{*}^{N}, \bar{a} \in \mathbb{P}^{N}$,

$$
\sum_{|\bar{s}| \leqslant 1}(-1)^{\bar{s}} f(\bar{x}+\bar{a} \bar{s})=(-1)^{N} a_{1} a_{2} \cdots a_{N} f_{12 \ldots N}(\bar{c}),
$$

where $\bar{x} \leqslant \bar{c} \leqslant \bar{x}+\bar{a}$. In view of the continuity of $f$ and $f_{12 \ldots N}$ on $\mathbb{P}^{N}$, conclusion (a) follows from (4.3).

(b) This follows from (a) by consideration of coordinate restrictions.

Note that (4.3) shows that if $(-1)^{N} f_{12 \ldots N}>0$ on $\mathbb{P}^{N}$, then $f$ is strictly $N$-monotone. Further, since full monotonicity on $\mathbb{P}^{N}$ implies full monotonicity on $\mathbb{N}^{N}$, (4.2) yields a simple test for full monotonicity on $\mathbb{N}^{N}$. Let $\mathbb{R}_{\bar{c}}^{N}:=\left\{\bar{x} \in \mathbb{R}^{N} \mid \bar{x} \geqslant \bar{c}\right\}$ and say that $f$ is $N$-monotone or fully monotone on $\mathbb{R}_{\bar{c}}^{N}$ if $f(\bar{x}-\bar{c})$ is similarly monotone on $\mathbb{R}_{\overline{0}}^{N}=\mathbb{P}^{N}$.

EXAMPLE 4.2. (a) Let $f(x, y, z):=\left(x^{2}+y^{2}+z^{2}\right)^{-q}$ for $q>0$ and $(x, y, z) \in \mathbb{R}_{\frac{3}{1}}^{3}$. Then

$$
f_{1}=-2 x q\left(x^{2}+y^{2}+z^{2}\right)^{-q-1}<0, \quad f_{12}=4 x y q(q+1)\left(x^{2}+y^{2}+z^{2}\right)^{-q-2}>0,
$$


and

$$
f_{123}=-8 x y z q(q+1)(q+2)\left(x^{2}+y^{2}+z^{2}\right)^{-q-3}<0
$$

on $\mathbb{R}_{\frac{3}{1}}^{3}$. By symmetry this suffices to show that $f$ is (strictly) fully monotone on $\mathbb{R} \frac{3}{1}$. The $N$-dimensional case can be treated similarly, as can the function in Example 2.1 (a).

(b) Consider the two-dimensional lattice sum

$$
\sum_{m, n=1}^{\infty}(-1)^{m+n}\left(\left(a_{1} m+b_{1} n\right)^{2}+\left(a_{2} m+b_{2} n\right)^{2}\right)^{-q},
$$

where $q>0$ and $a_{1}, a_{2}, b_{1}, b_{2}$ are real numbers. This corresponds to summing over the cone of vectors of the form $m \bar{a}+n \bar{b}$ for $m \geqslant 1, n \geqslant 1$, where $\bar{a}:=\left(a_{1}, a_{2}\right), \bar{b}:=\left(b_{1}, b_{2}\right)$. Let

$$
A:=a_{1}^{2}+a_{2}^{2}, \quad B:=b_{1}^{2}+b_{2}^{2}, C:=\bar{a} \cdot \bar{b}=a_{1} b_{1}+a_{2} b_{2},
$$

and

$$
D:=D(x, y)=\left(a_{1} x+b_{1} y\right)^{2}+\left(a_{2} x+b_{2} y\right)^{2} .
$$

Then $f(x, y):=D^{-q}(x, y)$ sațisfies

$$
\begin{aligned}
f_{1} & =-2 q(A x+C y) D^{-q-1}, \quad f_{2}=-2 q(C x+B y) D^{q-1}, \\
f_{12} & =4 q(q+1)(A x+C y)(C x+B y) D^{-q-2}-2 q C D^{-q-1} \\
& =2 q D^{-q-2}\left((2 q+1)\left(A x^{2}+B y^{2}\right) C+2 x y\left(q C^{2}+(q+1) A B\right)\right) .
\end{aligned}
$$

We see that $f_{12} \geqslant 0$ while $f_{1} \leqslant 0$ and $f_{2} \leqslant 0$ on $\mathbb{R}_{1}^{2}$ if and only if $C \geqslant 0$. Thus $f$ is fully monotone on $\mathbb{R}_{1}^{2}$ if and only if the angle between $\bar{a}$ and $\bar{b}$ is acute, and in this case the series in (4.4) converges in the sense of Theorem 3.1 or Theorem 3.2.

\section{References}

1. D. Borwein, J. M. Borwein, and K. F. Taylor, Convergence of lattice sums and Madelung's constant, J. Math. Phys., 26 (1985) 2999-3009.

2. T. J. Bromwich, An Introduction to the Theory of Infinite Series, 2nd ed., Macmillan, 1926.

3. M. L. Glasser and I. J. Zucker, Lattice sums, in Perspectives in Theoretical Chemistry: Advances and Perspectives, 5(1980) 67-139.

4. G. H. Hardy, On the convergence of certain multiple series, Proc. London Math. Soc., 2(1904) 24-28 and Proc. Cambridge Philos. Soc., 19(1917) 86-95. (See also Collected Papers, vol. 6, pages 5-10, and 629-639, Oxford, 1974.)

5. B. Meyer, On the convergence of alternating double series, this MonTHLy, 60(1953) 402-404.

6. F. Móricz, Some remarks on the notion of regular convergence of multiple series, Acta Math. Hungar., 41(1983) 161-168.

7. A. Wilansky, On the convergence of double series, Bull. Amer. Math. Soc., 53(1947) 793-799.

173.

\section{MISCELLANEA}

Too long for haiku!

Truly marvellous the proof

Of Fermat's theorem.

Robert J. MacG. Dawson

Corpus Christi College

Cambridge, United Kingdom

CB2 1RH 